\title{
CONSULE UERITATEM : CICÉRON, VARRON ET UN CHAPITRE DE L'HISTOIRE DE LA VÉRITÉ À ROME
}

\author{
Alessandro Garcea
}

Presses Universitaires de France | «Revue de métaphysique et de morale »

2008/1 n 57 | pages 93 à 110

ISSN 0035-1571

ISBN 9782130567929

Article disponible en ligne à l'adresse :

https://www.cairn.info/revue-de-metaphysique-et-de-morale-2008-1-page-93.htm

Distribution électronique Cairn.info pour Presses Universitaires de France.

(C) Presses Universitaires de France. Tous droits réservés pour tous pays.

La reproduction ou représentation de cet article, notamment par photocopie, n'est autorisée que dans les limites des conditions générales d'utilisation du site ou, le cas échéant, des conditions générales de la licence souscrite par votre établissement. Toute autre reproduction ou représentation, en tout ou partie, sous quelque forme et de quelque manière que ce soit, est interdite sauf accord préalable et écrit de l'éditeur, en dehors des cas prévus par la législation en vigueur en France. Il est précisé que son stockage dans une base de données est également interdit. 


\section{Consule ueritatem: Cicéron, Varron et un chapitre de l'histoire de la vérité à Rome*}

RÉSUMÉ. - Dans cet article, je me concentrerai sur le concept de ueritas en lien avec la grammaire et la rhétorique à la fin de la République romaine, au moment où elles devenaient des disciplines indépendantes. D'un point de vue intralinguistique et "autonome », la ueritas peut être conçue comme un système conventionnel de procédures de vérification établissant une correspondance entre chaque unité et les règles analogiques. Tel est l'arrière-plan sur lequel se découpent tant l'excursus grammatical de l'Orator de Cicéron que le De lingua Latina de Varron. Mais un autre point de vue, extralinguistique et hétéronome celui-là, figure également dans l'œuvre de ces deux auteurs. Cicéron élabore une théorie philosophique et rhétorique du uerisimile, afin de prétendre à la même force persuasive que celle que détiennent les choses elles-mêmes, autrement dit la réalité de l'évidence. Varron estime que la reconstruction d'un système totalisant, tel que celui du langage, gouverné par l'analogie, conduit à l'acquisition d'une vérité universelle qui peut être découverte par le biais de l'analyse morpho-étymologique des mots latins.

ABSTRACT. - In this article I will focus on the concept of ueritas in relation to grammar and rhetoric during the late Roman Republic, when they were becoming independent disciplines. From an intralinguistic and "auto-nomous » perspective, ueritas can be conceived as a conventional system of verificational procedures which establish the correspondence between each unit and the analogical rules. This is the background of both the grammatical excursus of Cicero's Orator and Varro's De lingua Latina. But another perspective, an extralinguisc and heteronomous one, is also present in the work of these authors. Cicero conceives a philosophical and rhetorical theory of uerisimile, in order to pretend to the same persuasive force which things themselves, i.e. the evidential reality, have. Varro thinks that the reconstruction of a totalising system, such as that of language, governed by analogy, leads to the acquisition of a universal truth which can be discovered via the morpho-etymological analysis of Latin words.

Unverborgenheit, «dévoilement », c'est la manière dont Martin Heidegger rend le grec alêtheia, mot traduit usuellement par «vérité », pour exprimer l'idée propre à l'esprit grec selon laquelle la vérité constitue une sorte de

\footnotetext{
* Nous tenons à remercier chaleureusement Lucia Calboli Montefusco et Sophie Aubert pour les suggestions et les remarques qu'elles ont bien voulu nous transmettre.
} 
révélation de la nature ${ }^{1}$ : ce n'est qu'après la contemplation de celle-ci - ou theoria - que l'homme développerait la capacité de créer un savoir qui porte sur l'agir - ou praxis -, la connaissance de la nature s'arrêtant ainsi devant l'impossibilité de tout changement par la technique, contrairement à ce que prétend la pensée moderne ${ }^{2}$. Dans cet article, nous nous proposons de réfléchir à quelques formes d'appropriation du concept grec de « vérité » par les Romains, ainsi qu' aux conséquences que ce phénomène d'acculturation a entraînées moins d'un point de vue inter-culturel qu'intra-culturel, à savoir par rapport à la constitution des disciplines de la grammaire et de la rhétorique à Rome. À cette fin, nous établirons une comparaison entre Cicéron et Varron qui, s'ils poursuivent le même effort de rationalisation en vue de la création d'un savoir organisé, se différencient radicalement par la manière dont ils entendent les fondements de leurs systèmes.

La partie de l'Orator consacrée aux questions grammaticales (§ 155-162) montre que Cicéron avait choisi de se mesurer avec ses adversaires néo-attiques sur un terrain technique, dont cependant il avait déjà affirmé le caractère étranger à l'éloquence au livre III du De oratore ${ }^{3}$. La position qu'il attribue à cet excursus

1. La reprise à Heidegger de ce concept ne nous astreint nullement à suivre ce philosophe dans sa radicalisation du thème de l'alêtheia, en opposition à une ueritas de l'imperium dans le monde latin. Pour une reconstitution de cette théorie, cf. notamment Mario RUGGENINI, « Veritas e $\dot{\alpha} \lambda \dot{\eta} \theta \varepsilon ı \alpha$. La Grecia, Roma e l'origine della metafisica cristiano-medioevale », Quaestio 1, 2001, numéro thématique : Heidegger e $i$ medioevali (Costantino Esposito \& Pasquale Porro eds.), pp. 83-112. Sur l'histoire de la vérité, notamment dans l'Antiquité classique, on se reportera, en dernier lieu, aux essais réunis par Markus ENDERs \& Jan SzAIF (eds.), Die Geschichte des philosophischen Begriffs der Wahrheit, Berlin/New York, de Gruyter, 2006.

2. Même en l'absence de citations ponctuelles, notre réflexion sur cette problématique a été profondément influencée par l'ouvrage très stimulant d'Umberto GALIMBERTI, Psiche e techne. L'uomo nell'età della tecnica, Milano, Feltrinelli, 1999, notamment le chapitre 38, « La verità come efficacia ».

3. Wilhelm Kroll (M. Tullii Ciceronis orator, Berlin, Weidmann, 1913, pp. 12-13) remarque à juste titre que l'analogie grammaticale et le néo-atticisme, s'ils ont comme caractéristique commune le fait d'être des doctrines dogmatiques, ne coïncident pas complètement. Son hypothèse, qui veut que l'Arpinate tire de Varron les arguments pour attaquer les partisans de l'école alexandrine, et notamment César (p. 138), a été doublement critiquée par Hellfried DAHLMANN (« Caesars Schrift über die Analogie », Rheinisches Museum 84, 1935, pp. 258-275 : 272 n. 1) : «Es ist mir unwahrscheinlich, dass Cicero gerade ein Stück aus Varro entlehnte, um ihm eine Aufmerksamkeit zu erweisen, in dem er jedenfalls nicht seine eigene Auffassung vertreten haben kann ; denn Varro ist Analogist. Die vermeintliche Berührungen sind auch nichtssagend. Weiter [...] dass er gerade Caesar treffen wollte, ist nicht gesagt. » Cela ne revient pas à considérer Cicéron comme un anomaliste car, comme le remarque Paul MoriLlon («Cicéron et les écoles grammaticales de son temps », in Jean Collart (éd.), Varron, grammaire antique et stylistique latine, Paris, Les Belles Lettres, 1978, 
dans la structure générale de l'œuvre confirme son avis sur le sujet : au lieu de réserver une section autonome à l'ars grammatica, l'Arpinate aborde les questions relevant de cette discipline dans le cadre de la mise en place des mots, exercice amplement dominé par le souci de l'euphonie ${ }^{4}$. Aussi remarque-t-il de nombreux cas où le recours à un critère de correction étymologico-formelle (ueritas) entre en contradiction avec la sensation acoustique produite par un mot. Résumons de manière schématique ses différentes prises de position ${ }^{5}$ :

1. La terminaison analogique - ōrum, pour les thèmes en -o-, est considérée comme étant la seule correcte ; toutefois, en dépit des attestations hétérogènes de Pacuvius ${ }^{6}$, de nombreuses locutions figées demandent le maintien de la terminaison originaire - um (par exemple, pro deum atque hominum fidem ou centuria fabrum), ce qui amène Cicéron à se conformer tout simplement à l'usage dans sa diversité (§ 156) :

« Je me rends compte de ce qui est correct [uerum] ; mais tantôt je m'exprime comme on le tolère, en disant soit pro deum, soit pro deorum, tantôt comme c'est obligatoire, en disant trium uirum, et non uirorum, sestertium, nummum, et non sestertiorum, nummorum, parce que dans ces cas l'usage est invariable. »

2. L'alternance entre formes verbales pleines et formes verbales réduites, les premières correctes, les secondes d'usage, est illustrée sur la base de l'usus scribendi de Térence, qui - de manière opportune pour Cicéron - emploie les deux ${ }^{7}$. De même, si la terminaison correcte pour la troisième personne du pluriel du parfait est - _runt et non - _re, l'usage ne saurait être réformé quand, pour des questions d'euphonie, il contredit cette norme, ainsi que le montre un exemple d'Ennius (§ 157) :

«Je ne critiquerai pas "D' autres ont écrit [scrips_re] sur le sujet" [Ennius ann. $213 \mathrm{~V}$. $=206 \mathrm{~S}$.] ; scripserunt à mon sentiment est plus juste [uerius], mais je me plie volontiers à l'usage, qui tient compte de l'oreille. »

3. Dans le cas du pronom idem, Cicéron, d'abord, constate que les nominatifs idem et eidem sont attestés respectivement chez Ennius et dans des inscriptions, puis, il prend en considération deux allomorphes avec $-s-$, dont le premier, isdem, révèle clairement son étymologie (du pronom is associé à la particule d'identité -dem), sans

pp. 253-262 : 257), « un anomaliste éviterait évidemment d'employer les mots ueritas ou recte pour qualifier des formes analogistes opposées à des formes anomales ».

4. Cf. Albert Yon, Cicéron. L’orateur, Paris, Les Belles Lettres, 1964, pp. LXVII-LXVIII.

5. Cf. également Alessandro GARCEA \& Angelo GiAVATTO, «Wahrheit und Sprachgebrauch : Zur Wiederverwendung zweier sprachwissenschaftlicher Begriffe zwischen Grammatik, Rhetorik und Philosophie in Rom », in Stephanos Matthaios \& Peter Schmitter (eds.), Linguistische und epistemologische Konzepte - diachron, Münster, Nodus, 2007, pp. 37-60 : 38-42.

6. Cf. Pacuvius trag. $80-81 \mathrm{R}^{3}=$ frg. $76,1-2 \mathrm{~S}$. ; inc. fab. $34 \mathrm{R}^{3}=264 \mathrm{~S}$.

7. Cf. orat. 157 : Térence Phorm. 383. 390 ; inc. auct. trag. frg. 194-195 R. ${ }^{3}$ (mais Térence selon Cicéron). 
toutefois qu'il puisse être adopté pour des raisons euphoniques, et le second, où le $-\bar{\imath}$ - est rendu par la diphtongue graphique, s'avère redondant $(\S 157)^{8}$ :

«"Le même [idem] champ a..." dit Ennius [ann. 477 V. ${ }^{2}=504$ S.], et on lit dans les temples "Le même [<e>idem] a approuvé" ${ }^{9}$, mais isdem aurait été plus exact [uerius], non pourtant eisdem qui est redondant ; isdem sonnait mal ; l'usage a obtenu qu'on fît une faute pour l'agrément de l'oreille. »

De la même manière, les formes étymologiquement transparentes postmeridianus, quadriiugae, mehercules, non scire, medidiem sont opposées aux formes d'usage pomeridianus, quadrigae, mehercule, nescire, meridies.

4. La préposition $a f$, qui pour Cicéron est à l'origine des allographes $a, a b, a b s$, ne peut être remplacée par aucune forme transparente sur le plan étymologique. Qui plus est, le critère euphonique a imposé l'élimination d' $a f$, pour ne retenir qu' $a, a b, a b s$ (§ 158):

«Particulièrement désagréable est la préposition af, qui ne subsiste plus maintenant que dans les livres de comptes, et même pas dans ceux de tout le monde; dans le reste de la langue elle a été modifiée : nous disons $a$-mouit et ab-egit et $a b s-t u l i t$, de sorte qu'on ne sait plus si la forme authentique [uerum] est $a$, ou $a b$, ou $a b s$. »

5. L'allongement vocalique dans les préverbes in- et con-, quand ils sont suivis de $s$ - et $f$ - à l'initiale du second membre du composé (par exemple, ìn-sanus, inn-felix, cōn-sueuit, cōn-fecit), représente un phénomène phono-syntaxique qui contredit l'analogie, mais qui ne doit pas être corrigé, car il tire son origine d'une raison euphonique $(\S 159)^{10}$ :

«Consulte la règle [ueritatem] de formation des mots : elle condamnera ; rapporte-t'en à l'oreille, elle approuvera ; demande-lui pourquoi, elle dira que cela lui plaît. Or le langage doit se plier au plaisir de l'oreille. »

6. Enfin, l'Arpinate rappelle comment il a abordé le problème de l'aspiration consonantique qui, pour lui, n'est pas propre au latin : d'abord, il ne mettait d'aspiration nulle part, afin de respecter la tradition linguistique de ses ancêtres ; puis, il a accepté de l'insérer dans les cas de pulchro-, Cethegus, Carthago, triumphus, tout en restant cantonné à l'usage quand il s'agissait de sepulcrum, corona, lacrima, pour des raisons euphoniques, et d'Orciuius, Mato, Oto, sur la base d'un critère esthétique $(\S 160)^{11}$ :

8. Le même problème est abordé par César anal. frg. 10 F. = $11 \mathrm{~K}$. (chez Charisius pp. 141,25142,3 B.).

9. Cf. CIL $1.2,638 ; 736 ; 745 ; 1739$ al.

10. Cf. aussi Aulu-Gelle 2,17, qui cite le passage de Cicéron ( $\$ 2$ ) et qui se demande pourquoi le préverbe pro ne suit pas le même comportement, quand il est suivi de $f(\S 3)$.

11. Cf. aussi Varron frg 280d-e F. = 80-81 G. \& S. (chez Charisius p. 93,3-9. 103,8-13 B.). 
«Moi-même, sachant que nos ancêtres ne mettaient d'aspiration nulle part que dans les voyelles, je prononçais pulcros, Cetegos, triumpos, Cartaginem. Une fois que, sur le tard, les reproches de mes oreilles m'eurent fait abandonner mon souci de la correction [ueritas], j'ai concédé au peuple le droit de fixer l'usage, et j'ai gardé ma science pour moi. Nous disons cependant Orciuios, Matones, Otones, Caepiones, sepulcra, coronas, lacrimas parce que le jugement de l'oreille le permet. »

C'est le seul exemple où la ueritas se justifie non en fonction de l'analogie ou de l'étymologie, mais par rapport aux habitudes des anciens; d'ailleurs, ce n'est que pour une partie limitée de cas que le principe de la suauitas entraîne un éloignement de la ueritas.

Les mots ueritas et uerum, dont nous venons de rappeler rapidement l'ensemble des occurrences dans l'excursus, désignent la correction sur les plans étymologique et morphologique ${ }^{12}$. Cette alliance de la formation des mots et de l'analogie correspond à la ratio $^{13}$, à l'aspect systématique de la langue, qui rend compte de la structure des unités lexicales. De ce point de vue, l'étymologie elle-même porte moins sur la définition du signifié que sur la restitution de la forme morphologiquement correcte et transparente d'une expression quelconque. De manière semblable, au sujet du même type de savoir, après avoir rappelé que Cicéron avait proposé la dénomination ueriloquium ${ }^{14}$ sans pourtant employer réellement ce néologisme, Quintilien affirmera (inst. 1,6,30. 32) ${ }^{15}$ :

«Parfois aussi, cette science tente de distinguer les formes barbares des formes corrigées : faut-il appeler la Sicile Triquetra ou Triquedra $[<$ quadrum] ? Faut-il dire

Comme le remarque Maria Luisa PORZIO GERNIA ( «Vicende storiche e culturali dell'aspirazione latina », Archivio glottologico italiano, 59, 1974, pp. 56-102 : 100), « di fronte al ripristino dell'aspirata nel II-I sec. a.C. è difficile non pensare ad un'influenza straniera; la restaurazione di $h$ - fu un'affettazione elegante ed erudita dei circoli imbevuti di ellenismo. [...] I Romani colti difendevano questa pronuncia corretta dell' $h$-, e dell'aspirazione in genere, dalla tendenza ad eccedere nell'aspirazione, che era un carattere dialettale e costituiva quella rustica asperitas e peregrina insolentia che Cicerone biasimava in Arrio [cf. de orat. 3,44 A.G.]».

12. Les interprétations de John Edwin SANDYs (Marcus Tullius Cicero. Ad Marcum Brutum orator, Cambridge, University Press, 1885 [Hildesheim, Olms, 1973], p. 172, qui traduit uerum par « grammatically correct according to the law of analogy ») et de Veli-Matti Rissanen («Some Aspects of Cicero's Conception and Use of Analogy», in Jyri Vaahtera \& Raija Vainio [eds.], Vtriusque linguae peritus. Studia in honorem Toivo Viljamaa, Turku, Turun Yliopisto, 1997, pp. 120125 : 124, qui remarque : "with the use of ueritas Cicero is referring to the strict rule of analogy ») ne s'avèrent pas complètement convaincantes.

13. Chez Quintilien (inst. 1,6,1-3), la ratio constitue le genre qui subsume l'analogia et l'etymologia : cf. Valeria LOMANTO, « Il sistema del sermo Latinus in Quintiliano », in Giorgio Bàrberi Squarotti et alii (eds.), Voce di molte acque. Miscellanea di studi offerti a Eugenio Corsini, Torino, Zamorani, 1994, pp. 237-256 : 240-246.

14. Cf. Cicéron top. 35 ; Benedetto Riposati, Studi sui topica di Cicerone, Milano, Vita e Pensiero, 1947, pp. 85-88.

15. Sur l'étymologie chez Quintilien, cf. Lomanto, « Il sistema », art. cit., pp. 244-246. 
meridies ou medidies ? et ainsi de suite pour d'autres mots qui obéissent à l'usage. [...] Je ne parle pas de ces problèmes mineurs, sur lesquels s'épuisent surtout les passionnés d'étymologie, qui, tenant à ramener à leur étymon [ad ueritatem] les termes qui ont subi une légère altération, recourent à des procédés variés et multiples, abrégeant ou allongeant, ajoutant ou retranchant, faisant permuter des lettres ou des syllabes. Il en résulte que des esprits tordus glissent à la fin dans les plus détestables fantaisies. »

Émanant de la même perspective rhétorique, cette convergence entre Cicéron et Quintilien montre que tous deux envisagent l'ars grammatica dans son aspect normatif, comme un savoir bien délimité, et par conséquent «auto-nome », qui s'applique au système fermé de la langue et qui permet d'établir des règles de fonctionnement censées à la fois confirmer les choix des locuteurs et corriger leurs habitudes erronées. Cela étant, comme les caractéristiques qui rendent un discours immédiatement compréhensible (explanatio) et proprement latin (Latinitas $)^{16}$ sont plutôt assurées par le respect de la consuetudo et, secondairement, de la suauitas ou euphonia ${ }^{17}$, ils considèrent que les analyses étymologiques et la constitution des paradigmes analogiques jouent un rôle secondaire dans l'arsenal de l'orateur ${ }^{18}$. Le lien étroit entre les trois principes auxquels ils accordent la primauté est explicitement déclaré au début de l'Orator $(\$ 80)^{19}$ :

"Quand il s'agit des mots propres et usuels (in propriis usitatisque uerbis), pris isolément, on approuve soit celui qui sonne le mieux (optime sonat), soit celui qui exprime le mieux l'idée (rem maxime explanat). »

16. Suivant un modèle aristotélicien (cf. rhet. 3,5), l'auctor ad Herennium considère la Latinitas et l'explanatio comme étant si étroitement liées qu'il les associe dans le concept d'elegantia $(4,17)$. $\mathrm{Au}$ livre III du De oratore, Cicéron mentionne lui aussi les uirtutes du Latine dicere et du plane dicere en lien étroit l'une avec l'autre (§ 38-51: cf. Anton D. LEEMAN, Harm PINKSTER \& Jakob Wisse, M. Tullius Cicero, De Oratore Libri III, Kommentar, 4, Buch II, 291-367 ; Buch III, 1-95, Heidelberg, Winter, 1996, pp. 179-183). Sur le rapport de la Latinitas au concept de « propre », cf. Marc Baratin, La naissance de la syntaxe à Rome, Paris, Minuit, 1989, pp. 343-360.

17. De la même manière, Quintilien opposera la ueritas ou ratio qui correspond à la forme des préfixes pris isolément à leur modification à l'ouïe lorsqu'ils entrent en composition : «On demande souvent si, en écrivant les prépositions, il convient de se conformer au son que l'on entend, lorsqu'elles sont en composition, ou quand elles sont isolées comme, par exemple, quand je prononce optinuit - la raison demande en effet un $b$ comme deuxième lettre, tandis que l'oreille entend plutôt un $p$ - et immunis, où la lettre $n$, que réclame la vérité, est transformée en $m$ sous l'influence dominante de la syllabe qui suit » (inst. 1,7,7).

18. Sur le critère de la consuetudo chez Cicéron, cf. G. LOPEZ, « Consuetudo elegans Latini sermonis (Cic. or. 153). Appunti per una ricostruzione del concetto ciceroniano di linguaggio », Quaderni dell'istituto di lingua e letteratura latina 4, 1982, Università di Roma « La Sapienza », Facoltà di Magistero, pp. 49-55.

19. Cf. aussi orat. 150. 162-163 ; de orat. 3,150. 171-172 ; Quintilien inst. 1,5,4 ; 8,3,16-17 ; Giovanna GARBARINO, «Verba poetica in prosa nella teoria retorica da Cicerone a Quintiliano », Memorie dell'Accademia delle Scienze di Torino 5.2, 1978, pp. 141-235 : 171-172. 201-202. 
Ce cadre théorique justifie l'intolérance de Cicéron pour l'école d'Alexandrie et le ton polémique de son excursus grammatical: la ueritas que l'Arpinate évoque à plusieurs reprises dans ce long passage correspond au critère normatif ou ratio propre à l'ars grammatica, dont l'efficacité en termes pragmatiques est largement dépassée par une autre ueritas, celle de la persuasion, propre à la rhétorique. Celle-ci emploie les mots comme des instruments pratiques permettant de fonder un horizon épistémologique qui s'approche du vrai et qui est fait valoir comme tel, afin de déterminer une réponse comportementale du public $^{20}$.

La puissance de cette autre vérité est affirmée à plusieurs reprises dans les discours cicéroniens, mais c'est notamment dans l'exorde du Pro Cluentio que l'on retrouve des précisions d'ordre gnoséologique. S'adressant aux juges, l'orateur leur demande, d'abord, de ne pas se fonder sur leurs a priori; puis, de permettre à la vérité du plaidoyer qu'ils entendront de remplacer leurs fausses opinions (\$ 6) :

« Ma seconde requête, c'est, si vous avez déjà retenu une opinion [opinionem... comprehendistis] dans votre esprit, quand la réflexion la ruinera [si ratio conuellet], que le discours la fera chanceler [si oratio labefactauit], qu'enfin la vérité l'extirpera [si denique ueritas extorquebit], de ne pas résister, de la laisser fuir de votre pensée avec plaisir ou du moins avec sérénité ${ }^{21}$. »

Curieusement, la terminologie de ce passage ressemble beaucoup à celle de l'exposé varronien des Academica posteriora sur la théorie de la connaissance chez Zénon, où la représentation « compréhensive » d'un objet est censée constituer à la fois le critère de vérité stoïcien et une sorte d'indifférent. L'acceptation de toute phantasia dépendant en effet de la qualité de l'hégémonique du sujet sentant, l'ignorance (inscientia = agnoia) du sot ne pourra que produire des

20. Cette interprétation rejoint les résultats présentés par M. Carlos Lévy dans une conférence intitulée «Rome ou l'oubli de l'ontologie » (ENS - Paris, 5 octobre 2007) : Cicéron ne serait pas intéressé par l'ontologie de la parole en elle-même ; il se concentrerait plutôt sur la uis de celle-ci.

21. Ainsi, très justement, Sandra Citroni MarchetTi ( Lo spazio straniato. Percorsi psicologici e percezione del tribunale nelle orazioni di Cicerone pro Fonteio, pro Q. Roscio comoedo, pro Cluentio. II , Materiali e Discussioni 36, 1996, pp. 33-71: 39-41) remarque : « È, questo, il processo psichico con cui un soggetto si libera dell'opinio che è causa del suo errore e della sua passione. Ma possiamo chiederci se in questo caso la ratio e la ueritas non siano subordinate al discorso, alla oratio : se l'oratore non intenda che debbano essere la "sua" ragione e la "sua" verità. [...] L'invito a "non ribellarsi" non domanda la rinuncia ad un pensiero falso per una sapienza oggettiva, ad un pregiudizio per una salda informazione, quanto piuttosto l'allentamento del controllo nei confronti delle parole dell'oratore. [...] Il tribunale è stato invitato a trasformarsi, possiamo dire, in puro uditorio attraverso la sospensione del pensiero critico : di ciò appunto che dovrebbe contraddistinguerlo in quanto tribunale. » 
opinions $($ opiniones $=$ doxa $)$ instables, non la science $($ scientia $=$ epistêmê $) \mathrm{du}$ sage, quelles que soient les perceptions reçues $(a c .1,41)^{22}$ :

"Quant à ce qui était "compris" [comprensum] par le sens, il l'appelait sensation et, si la "compréhension" était telle qu'elle ne pouvait être ruinée par la raison [conuelli ratione], il l'appelait science; dans le cas contraire, il la nommait ignorance. De cette dernière, disait-il, surgit l'opinion (opinio), qui est faible et qui participe à la fois de l'erreur et de l'ignorance. » (trad. C. Lévy)

Cela étant, le passage du Pro Cluentio mêle le terme oratio à la ratio et à la ueritas du processus gnoséologique censé faire disparaître les opinions des juges et conditionner leur assentiment. La vérité de l'éloquence assume ainsi un caractère d'objectivité indéfectible : sans être une représentation « compréhensive » de la réalité sensible, elle en prend quand même toute l'apparence dans les limites de l'univers clos du discours. Preuve en est la réponse de Quintilien à l'accusation selon laquelle la rhétorique ne serait pas une ars car elle se fonde sur des opinions fausses (inst. 2,17,19. 21) ${ }^{23}$ :

« Je veux bien admettre, pour ma part, que la rhétorique présente parfois le faux à la place du vrai, mais je ne concéderai pas, pour autant, qu'elle se fonde aussi sur le faux, car il y a une grande différence entre avoir soi-même une opinion et tenter de l'inspirer aux autres. [...] Quand Cicéron, à propos du procès de Cluentius, s'est vanté d'avoir enténébré l'esprit des juges, n'a-t-il pas vu clair lui-même ?»

La démarche qui consiste à remplacer les opinions du public et celles des juges par ce type de vérité rhétorique est une constante des discours cicéroniens, notamment quand il s'agit de contrecarrer la parole des adversaires. Dans ces cas, les catégories du vrai et du vraisemblable, des faits et des habitudes, de la raison et des opinions sont astucieusement superposées :

1. dans le Pro Sexto Roscio, Cicéron attaque Erucius : «Tu vois, Erucius, combien ton argumentation est en désaccord avec les faits et avec la vérité [ab re ipsa atque

22. Sur ce passage extrêmement important pour l'histoire du stoïcisme, cf. Carlos LÉvy, Cicero Academicus. Recherches sur les Académiques et sur la philosophie cicéronienne, Paris, de Boccard \& Rome, École française de Rome, 1992, pp. 224-231 ; pour les textes grecs parallèles : cf. Zénon SVF 1,62 et 68 .

23. Cf. Giancarlo Mazzoli, «Quintiliano e la Pro Cluentio », in Napoleone Stelluti (ed.), Pro Cluentio di Marco Tullio Cicerone. Atti del convegno nazionale (Larino 4-5 dicembre 1992), Larino, Amministrazione comunale, 1997, pp. 97-106, et, plus généralement, pour un aperçu récent et convaincant des enjeux rhétoriques du Pro Cluentio : Emanuele NARDUCCI, «Cronaca criminale e letteratura nella pro Cluentio », in Emanuele Narducci \& Marco Fucecchi, Marco Tullio Cicerone. Difesa di Cluenzio, Milano, Bur, 2004, pp. 5-37, notamment pp. 34-37 « L'avvocato e il filosofo ». 
a ueritate $]^{24}$. [...] Toi-même, tu n'es pas sans te rendre compte de la fausseté de tout ce que tu dis ; mais tu te trouves tellement à court d'arguments que tu te juges forcé d'aller à l'encontre non seulement de ce que nous disons, mais même de la nature des faits [contra rerum naturam], de la coutume des hommes [contraque consuetudine hominum], des opinions reçues par tout le monde [contraque opiniones omnium] » $(\S 44-45)^{25}$;

2. dans l'exorde du Pro Fonteio, l'orateur s'exclame: «Mais qu'est-ce que cette accusation? Qu'y a-t-il en elle qui s'accorde avec la raison [rationem], avec la coutume [consuetudinem], avec la vraisemblance [similitudinem ueritatis] ? Or, ce qui répugne à la raison [ratio], à la coutume [consuetudo], à la nature même de la chose [rei natura], faut-il le croire ?» (frg. 4 Boulanger).

Le même type d'antithèse figure quand il s'agit de discréditer les témoins des adversaires :

3. dans le Pro Quinctio, au moment de l'appel des témoins à charge, l'orateur tient à souligner le rôle extrêmement ambigu de ceux-ci, car «s'ils respectent la vérité, ils pourront affirmer leur importance, mais, s'ils négligent la vérité, tout le monde comprendra que l'autorité d'un témoin est une aide utile pour établir la vérité, mais non pour affirmer le mensonge » (\$75); puis, comme il dispose d'éléments en sa faveur qu'il tient pour incontestables, Cicéron défie ses illustres adversaires de le contredire : «Oui, quand même il serait permis à tous les Crassus de paraître ici avec tous les Antoines, voudrais-tu toi-même, Lucius Philippus, dont la gloire florissait à côté de la leur, voudrais-tu plaider cette cause avec Hortensius : il est de toute nécessité que j'aie le dessus ; car tous les éléments de succès ne résident pas, comme vous le pensez, dans l'éloquence : il est aussi des cas où la vérité est tellement évidente [ita perspicua ueritas] que rien ne saurait l'affaiblir ${ }^{26}(\S 80)$;

24. Gustav Landgraf (Kommentar zu Ciceros Rede pro Sex. Roscio Amerino, Leipzig, Teubner [Hildesheim, Olms, 1978], 1914², ad loc.) penche pour un hendiadyin : «von dem wirklichen Sachverhalt », auquel s'opposent des locutions du type uerba et ineptiae.

25. Il est important de remarquer que cette déclaration apparemment innocente correspond en réalité à une stratégie rhétorique d'appropriation de la thèse de l'adversaire pour la renverser : « The prosecution had hargued that Roscius' father had relegated him in Rome [...], so Roscius had his father killed to avoid the risk of being desinherited [...]. Cicero counters that to be put in charge of rural properties was, both in this particular case and in Roman tradition generally, a mark of favor [...]. Hence Roscius had no motive for the crime. On the contrary, his so-called "relegation" proved his good relationship with his father » (Andrew M. RIGGSBY, « Appropriation and Reversal as a Basis for Oratorical Proof », Classical Philology 90, pp. 245-256 : 245-246). Plus généralement, dans ce procès, le stéréotype de l'opposition entre la cité et la campagne est utilisé aussi bien par l'accusation que par la défense, dans deux buts opposés : cf. Ann VASALY, « The Masks of Rhetoric : Cicero's Pro Roscio Amerino », Rhetorica 3, 1985, pp. 1-20.

26. La faiblesse des arguments de Cicéron amène Thomas E. KinsEY ( Cicero, Hortensius and Philippus in the Pro Quinctio », Latomus 29, 1970, pp. 737-738: 737) à cette conclusion : «One part of Cicero's picture of himself as a diffident orator taking courage from the strenght of his case seems then of doubtful sincerity and the other part may be equally dubious. Perhaps we should bear in mind the possibility that the real Cicero was an orator confident enough of his own powers 
4. l'apport très limité des témoins est également critiqué dans le Pro Sulla, en lien avec la quaestio per tormenta ou témoignage rendu sous la torture : «... dans ces tortures c'est la douleur qui règne ; tout dépend du tempérament physique et moral de chacun ; le magistrat instructeur agit en maître, la passion fausse les témoignages, l'espérance les altère, la crainte leur enlève toute valeur, si bien que parmi tant d'entraves la vérité ne peut se faire jour [nihil ueritati loci relinquatur] » (§ 78);

5. dans le Pro Caelio l'orateur soumet préalablement à la discussion la valeur des dépositions qui pourraient nuire à sa thèse : «En ce qui me concerne, je veux vous [scil. les juges] soustraire à l'influence des témoins et, dans ce procès, je ne laisserai pas la vérité, que rien ne peut modifier [ueritatem quae mutari nullo modo potest], dépendre de leur bon vouloir, qu'il est très facile de manœuvrer, qu'un rien peut fléchir et orienter. C'est avec des arguments rationnels que nous plaiderons, avec des preuves matérielles plus claires que le jour que nous réfuterons l'accusation ; aux faits nous opposerons les faits, aux motifs les motifs, aux raisons les raisons » (\$22); puis il réaffirme les mêmes propos au moment où les témoins font leur apparition dans le tribunal : «Ô pouvoir éminent de la vérité [o magnam uim ueritatis], qui, malgré l'ingéniosité des hommes, leur habileté, leur adresse et tous les subterfuges qu'ils inventent, peut aisément se défendre d'elle-même ! ( $($ 63) ; «Aussi bien, dans cette cause, a-t-on négligé les preuves, les conjectures, les indices, qui font ordinairement éclater la vérité [quibus ueritas inlustrari solet], pour s'en remettre aux seuls témoins » $(\S 66)^{27}$.

Les passages du Pro Caelio montrent clairement que l'attaque contre les témoins vise à faire la distinction entre, d'une part, les preuves qui ne dépendent pas de l'ars rhétorique (pisteis atekhnoi ou probationes inartificiales), notamment les dépositions des témoins, et, d'autre part, les conclusions tirées du cas en question via les instruments de la rhétorique (pisteis entekhnoi ou probationes artifica$l e s)^{28}$. C'est sur ces éléments que l'orateur s'appuie pour démontrer sa « vérité », et notamment sur l'êthos, tenu pour preuve irréfragable à l'instar des signa et des argumenta, en dépit de son caractère subjectif :

6. dans le Pro Plancio, la présence d'hommes illustres qui sont venus assister l'accusé est considérée comme un signe de vérité indéniable qu'aucun discours ne peut altérer : «Ce sont là, juges, des preuves réelles, explicites, ce sont des indices de droiture, qui ne sont pas fardés pour faire bon effet au tribunal [signa probitatis non fucata forensi specie], mais portent gravées les marques authentiques de la vérité [domesticis inusta notis ueritatis] » (\$29);

even at this stage to be ready to try to make his mark by taking on at short notice [...], in a poor case one of the leading orators of the day. »

27. Le lien entre l'aspect logico-déductif des preuves techniques et la sagesse des juges est bien étudié par H.-J. HARTUNG, « Religio und sapientia iudicum. Einige grundsätzliche Bemerkungen zu einem Geschworenenspiegel in Ciceros Reden », Hermes 102, 1974, pp. 556-566 : 563-565.

28. Pour cette distinction, et pour la taxinomie complexe qui en découle, cf. Heinrich LAUSBERG, Handbook of Literary Rhetoric, tr. angl. Leiden, Boston \& Köln, Brill, 1998 (München, Hueber, $1973^{2}$ ), \& 350-426. 
7. de même, dans le Pro Scauro, Cicéron loue la valeur des preuves techniques que sont pour lui le caractère et l'auctoritas : «Une preuve, en revanche, celle qui résulte d'un fait - et c'est là seulement ce qui mérite le nom de preuve -, est la voix de la réalité [rerum uox], la marque de la nature [naturae uestigium], le signe de la vérité [ueritatis nota] ; quelle qu'elle soit, elle reste nécessairement immuable, car elle n'est pas façonnée par un orateur, il la prend toute faite. C'est pourquoi, si j'étais vaincu en une accusation de cette sorte, je m'avouerais vaincu et je m'inclinerais ; je serais en effet vaincu par la réalité $[\mathrm{re}]$, vaincu par la cause [causa], vaincu par la vérité $[$ ueritate $] \gg(\S 16-17)^{29}$.

Dans cette optique, le rôle de l'orateur qui se sert des pisteis entekhnoi se réduit à celui de quelqu'un qui se limite à traduire, en l'exprimant linguistiquement, la réalité « authentique » des choses. Cette conception sous-tend la défense de Roscius qui, très apprécié comme acteur, hors plateau ne saurait jamais dissimuler la vérité, car il «réunit en sa personne plus de loyauté encore que de talent (plus fidei quam artis), encore plus d'amour de la vérité que de science de son art (plus ueritatis quam disciplinae) » $(Q . \text { Rosc. 17 })^{30}$. Le fait qu'il ait transigé séparément avec Flavius après avoir chargé Fannius de diriger le procès ne paraît pas très grave si l'on n'exagère pas, par les paroles et par l'opinion (oratione et opinione), les avantages médiocres, dont témoignent les faits et la réalité (re et ueritate), qu'il avait obtenus (\$33). L’orateur se déclare ainsi sous le pouvoir de la vérité : «C'est la vérité elle-même qui a usé sur moi de son droit de mainmise (ipsa mihi ueritas manum iniecit) et qui me contraint d'insister quelque temps sur cette question, de m’y arrêter» (§ 48). Les accusations de Fannius contre Roscius sont ainsi destituées de valeur car, comme elles sont fausses sur le plan de la vérité des choses, elles ne sont pas non plus crédibles sur le plan de la raison : quod cum est ueritate falsum, tum ratione quoque est incredibile (\$ 50).

Les mêmes principes sont affirmés dans le cadre de la réflexion cicéronienne du De oratore $^{31}$. Dans l'éloge de l'éloquence par Antoine au livre II, la mise

29. Comme le remarque James M. MAY (Trials of Character. The Eloquence of Ciceronian Ethos, Chapel Hill \& London, North Carolina Press, 1988, p. 120), ces mots s'appliquent au recours, de la part de Cicéron, aux «character witnesses »: «Appeal to the character and authority of such witnesses and their explicit identification with his client is again the favoured technique. [...] These are the kinds of proofs, Cicero argues, whose source lies in character and whose authority reside in character, that are incontrovertible, fashioned in flesh not words. »

30. Cf. Citroni MARChetTi («Lo spazio », art. cit., p. 41) : «La caratterizzazione di Roscio si basa su due serie di requisiti (fides/ars, ueritas/disciplina, abstinentia/artificium) : mentre la prima riguarda il uir, la seconda riguarda l'histrio : ma proprio quest'ultima rappresenta (illusoriamente, paradossalmente) la base su cui poggia la prima serie. »

31. Le seul qui considère le concept de vérité comme l'apport vraiment novateur de Cicéron à la rhétorique est Karl BüCHNER («Zur antiken Vorstellung vom Redner», in ID., Studien zur römischen Literatur, IX, Römische Prosa, Steiner, Wiesbaden, 1978, pp. 26-43), qui toutefois ne prend pas en compte les discours de l'Arpinate et, au sujet de la vérité elle-même, affirme : «Darum 
en parallèle de l'orateur et de l'acteur aboutit à une distinction essentielle : le premier n'imite pas la réalité comme le fait le second, mais il la «prend en charge », il la prend sur lui pour donner un ancrage à sa parole ( $\$ 34$ qui actor imitanda quam orator suscipienda ueritate iucundior?). Au tout début de la section sur l'actio du livre III, remarquant l'importance des gestes et de la voix, Crassus déplore que les acteurs, qui se limitent à imiter la vérité, se soient emparés de ces techniques, susceptibles de rentrer dans l'arsenal des orateurs, qui, eux, représentent la réalité elle-même ( $\$ 214$ ueritatis ipsius actores). En effet, l'éloquence a ceci de propre qu'elle permet de mettre en forme et d'organiser, en la rendant énonçable et par conséquent efficace, toute vérité potentielle que l'orateur est prêt à faire sienne ${ }^{32}$.

L'ensemble des passages que nous avons rappelés jusqu'ici s'avère parfaitement cohérent avec la valeur que la ueritas prend dans les traités philosophiques de Cicéron, si bien qu'on pourrait estimer que celui-ci était parvenu à délimiter le savoir propre à la rhétorique précisément grâce aux catégories que lui fournissait la réflexion néoacadémicienne en la matière. Comme l'a très bien démontré Carlos Lévy, la spéculation de Carnéade autour du concept de pithanon consistait à fonder l'agir sur le « sentiment de vérité que donnent certaines représentations, mais sans pour autant en tirer des conclusions quant

kann man actor ueritatis auch mit Gestalter der Wahrheit übersetzen, wofern man sich wie Cicero bewußt ist, daß sie sich zwar vom ewigen Gesetz der richtigen Vernunft herleitet, aber in den Situationen immer neu zu finden und durchzusetzen ist. » Cf. également l'expression proverbiale simplex oratio ueritatis caractérisant la célèbre défense du stoïcien Rutilius Rufus (de orat. 1,229 \{ratio L, Lehmann \& Pinkster : ratio recc., edd.\} ; sur ce passage, cf. Carlos LÉvy, « Cicéron critique de l'éloquence stoïcienne », in Lucia Calboli Montefusco (ed.), Papers on Rhetoric III, Bologna, Clueb, pp. 127-144 : 131-134 ; Gabriella MoRETTI, « Suscitare o no le passioni ? Il ruolo di Publio Rutilio Rufo », in Lucia Calboli Montefusco (éd.), Papers on Rhetoric IV, Roma, Herder, pp. 205-222), ainsi que, par rapport au témoignage de Lucius Lucceius dans le procès contre Caelius, haec [sc. L. Luccei] una uox ueritatis (Cael. 55). La formulation n'est pas nouvelle : cf. Anton D. Leeman, Harm Pinkster \& Hein L.W. Nelson, M. Tullius Cicero, De Oratore Libri III, Kommentar, 2, Buch I, 166-265; Buch II, 1-98, Heidelberg, Winter, 1985, p. 150.

32. Il faut également mentionner de orat. 2,94, passage consacré aux orateurs qui sont dotés de talents différents mais qui néanmoins poursuivent la même représentation réaliste (imitatio (im $_{1}$ ) des choses qu'est la qualité maîtresse de leur modèle (imitatio $)_{2}$ ), Isocrate. L'interprétation de cet exemple, encore jugé par LeEman, Pinkster \& Nelson (M. Tullius Cicero, op. cit., ad loc.) comme «befremdlich », demeure très controversée. Alan E. Douglas (« The Intellectual Background of Cicero's Rhetorica. A Study in Method », Aufstieg und Niedergang der römischen Welt 1.3, 1973, pp. 95-138: 114) songe à « an odd echo of the language of art-criticism in which the achievement of ueritatis imitatio marks the high peak »; Elain FANTHAM, d'abord, voit une allusion à la vie réelle : «The orator deals with actuality, with immediate, current events » («Imitation and Evolution. The Discussion of Rhetorical Imitation in Cicero De Oratore II 87-97 and Some Related Problems of Ciceronian Theory », Classical Philology 73, 1978, pp. 1-16: 9-10) ; puis propose, parmi les traductions possibles, « in the same style possessing authenticity and plausibility » (« On the Use of Genus-Terminology in Cicero's Rhetorical Works », Hermes 107, 1979, pp. 441-459: 445), mais on ne saurait expliquer la raison pour laquelle le style d'Isocrate est censé être particulièrement réaliste. 
à leur conformité à la réalité ». De cette manière, le philosophe « conciliait le sentiment de croyance et le travail de la raison, chargée par lui non d'établir une vérité absolue, mais de vérifier la cohérence de la chaîne des représentations ${ }^{33}$. Cicéron, qui connaît également l'importance du critère pratique de l'eulogon chez Arcésilas ${ }^{34}$, aboutit à une synthèse de ces principes dans la catégorie du probabile, mot qui fait référence «au sens commun, à la raison et, en définitive, à la vérité, même s'il n'en constitue qu'une approche incertaine » (ibid., p. 288), et qui peut être considéré comme synonyme de uerisimile ${ }^{35}$.

L'importance de ce concept pour l'orateur est capitale. Au début du livre III du De oratore, Crassus exprime sa préférence pour la disputatio in utramque partem académicienne et précise que, à l'aide de la philosophie, les meilleurs esprits sont capables de remarquer ce qu'il y a de vraisemblable dans chaque question (id quod in quoque ueri simile est), pour ensuite le mettre en forme grâce à l'éloquence $(\S 79)^{36}$. De même, au livre II du De officiis, l'auteur tient à distinguer le juge, qui doit avoir comme repère la vérité objective des faits (uerum), de l'orateur, qui plaide pour ce qui est vraisemblable, pas forcément vrai (\$ 51 ueri simile, etiam si minus sit uerum $)^{37}$. Ainsi devient possible une conception de la ueritas comme recréation, comme construction, par l'orateur, d'un univers délimité et, en tant que tel, ayant toutes les caractéristiques du vrai. Loin de permettre l'accès à une connaissance totalisante, le probabile constitue un instrument pour réaliser la persuasion, car grâce à lui la parole - tenue pour ni fausse, car elle ressemble au vrai, ni complètement vraie - devient un moyen opérationnel s'adaptant à toute situation. Une fois

33. Cf. LÉvy, Cicero, op. cit., pp. 284-285.

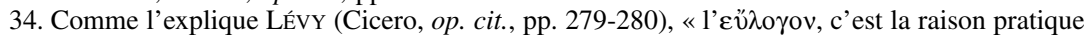
envisagée non pas du point de vue de sa capacité à accéder à la perfection morale, mais dans sa confrontation avec les incertitudes nées de l'ignorance de la trame du destin ».

35. Cf. notamment inu. 1,46. La même doctrine est appliquée à la narratio (inu. 1,29) ; cf. aussi rhet. Her. 1,2,3, en lien avec l'inuentio.

36. Sur ce sujet, notamment en lien avec le caractère volontaire des passions de l'orateur, cf. Emanuele NARDUCCI, « Gli arcani dell'oratore », in Emanuele Narducci et alii, Marco Tullio Cicerone. Dell'oratore, Milano, Bur, 1994, pp. 83-110, qui remarque : « Le passioni dell'oratore si presentano come qualcosa di ben diverso da banali perturbazioni dell'animo; si tratta piuttosto di passioni suscitabili a piacimento, i cui panni l'avvocato può indossare o smettere a seconda delle esigenze della causa, e senza che per questo gli si possa rimproverare una bassa finzione : perché si tratta sempre di emozioni reali, anche se sapientemente pilotate » (p. 95).

37. Cet extrait est attribué à Panaetius frg. 95 v. S. = test. 117 A. Sur cette section du De officiis, cf. Giovanna Garbarino, «Il concetto etico-politico di gloria nel De officiis di Cicerone », in Massimiliano Pavan \& Umberto Cozzoli (eds), Tra Grecia e Roma. Temi antichi e metodologie moderne, Roma, Istituto Poligrafico e Zecca dello Stato, 1980, pp. 197-204. Selon Francesca ALESSE, Panezio di Rodi. Testimonianze, Napoli, Bibliopolis, 1997, p. 252, Panaetius reprendrait un thème qui remonte à Platon pour faire le lien entre l'activité de ceux qui défendent l'intérêt individuel et la sensibilité commune. 
perdu le statut de cachette d'une vérité éternelle qui doit être dénichée, elle déploie sa puissance dans l'orientation des décisions d'autrui ${ }^{38}$.

Il est intéressant de comparer l'édifice épistémologique cicéronien avec les propositions de Varron en matière de langage. Même s'il aborde des problèmes identiques à ceux de l'excursus de l'Orator, dans son appropriation du concept de vérité, celui-ci s'efforce de concilier la raison propre à un savoir circonscrit comme la grammaire avec l'idée d'un principe unique qui constitue le fondement d'un savoir universel.

Ainsi, au livre IX du De lingua Latina, il affirme en préambule l'existence du système rationnel qu'est la langue; puis, il identifie cette ratio avec la consuetudo qua oportet uti, ce qui permet à cette forme de «bon usage » de représenter un repère sûr pour tous les locuteurs $(\S 8)$. Par conséquent, s'ils sont fléchis d'une manière non conforme à la bonne consuetudo, il faut resituer les mots d'un énoncé dans un rapport correct avec les paradigmes qui sont à la fois utilisés par tous les locuteurs et conformes à la ratio. Celle-ci, en tant que système d'expression potentiel fondé sur des corrélations analogiques, s'avère indépendante de l'utilisation effective des éléments linguistiques par la parole. Ainsi, à la fin du livre IX, en lien avec le problème des terminaisons parallèles $-i$ et $-e$ à l'ablatif, Varron remarque que si l'on choisit la forme non correcte, à savoir celle qui ne rentre pas dans les paradigmes réguliers, le modèle d'ordre préexistant ne s'en retrouve pas pour autant menacé ; il est en revanche confirmé lorsque l'on utilise la forme correcte (ling. 9,112):

«Si quelqu'un dit à l'ablatif monti, fonti et si quelqu'un d'autre dit monte, fonte - et le même discours pourrait s'appliquer à tous les mots ayant des variantes -, l'une étant la terminaison correcte [uerum], l'autre la fausse [falsum], celui des deux qui se trompe n'infirme pas le rapport analogique, alors que celui qui utilise la forme correcte le confirme. Et de même que celui qui se trompe sur les variantes d'un mot n'infirme pas le critère proportionnel quand il choisit la forme fausse, de même, avec les mots qui n'ont pas de variantes, si quelqu'un estime qu'il faille dire autrement de ce qu'il faut, il n'infirme pas pour autant la science du langage, mais il met à nu son ignorance. »

38. Comme le remarque fort justement NARDUCCI («Gli arcani », art. cit., p. 99), « ... tra il probabilismo del filosofo e quello dell'avvocato resta, comunque, una differenza significativa : il primo si preoccupa di fare emergere la verità, per quanto una verità non assoluta, e continuamente revocabile in discussione ; il secondo mira principalmente a una persuasione che gli concederà la vittoria ». 
Deux types de transgressions de la ueritas sont distingués: celles qui sont enracinées dans l'usage, et qui paraissent tolérables aux anomalistes, et les plus récentes, encore senties comme telles, sur lesquelles les anomalistes estiment possible d'intervenir ( $§ 10)$. Pour sa part, Varron propose d'éliminer tout de suite les formes fautives entrées récemment dans l'usage, et dont la substitution ne heurte pas la sensibilité des locuteurs, mais en même temps proscrit résolument le recours aux formes inexactes déjà employées depuis longtemps (§ 11). Pour étayer sa position, il institue une comparaison entre le progrès dans le domaine de la peinture et l'esprit intransigeant de l'analogiste par excellence, Aristophane de Byzance ( $\$ 12)$ :

« S'il ne faut pas reprocher aux peintres Apelle et Protogène, ainsi qu'à d'autres artistes excellents, de ne pas avoir suivi la manière de Mycon, de Dioris, d'Arimna, et d'autres encore plus anciens, faut-il désapprouver Aristophane car parfois il a préféré la forme étymologiquement correcte [ue[te]ritatem] à celle d'usage ${ }^{39}$ ? »

Dans une liste d'améliorations apportées à un précédent état de choses, notamment dans la peinture, dans la science militaire, dans le droit et dans l'école ( $\$ 12-15)$, la mise en parallèle de la peinture et de la grammaire s'avère particulièrement fructueuse. En effet Apelle et Protogène, les deux artistes rivaux de la fin du $\mathrm{IV}^{\mathrm{e}}$ siècle, figurent aussi dans le célèbre excursus du Brutus cicéronien où l'évolution des arts est mesurée par le critère de l'imitari ueritatem ${ }^{40}$. Censés représenter l'aboutissement d'un long parcours visant à produire une

39. Comme nous le montrerons plus loin, la traduction de ueritas par « forme étymologiquement correcte » correspond à l'esprit du De lingua Latina, où la correction morphologique présuppose la fidélité à l'origo du mot. Cf. LomANTO, « Il sistema », art. cit., pp. 244-246.

40. Cet excursus a pour but de montrer que, tout comme personne ne contesterait que l'histoire de la sculpture et celle de la peinture soient constituées d'une série d'étapes successives, dont chacune est marquée par une maîtrise plus développée de la technique, la norme nihil est ... simul et inuentum et perfectum (\$ 71) s’applique également à la littérature et à l'art oratoire. Les $\S 70-76$ sont structurés selon une série de passages analogiques : art oratoire - arts figuratifs [(a) sculpture - (b) peinture] - poésie [(a) épique grecque - (b) épique romaine] - art oratoire. Le même genre de structure figure au début du livre III du De oratore, où Crassus considère la possibilité de créer une théorie générale de la forme artistique, au-delà de l'antinomie entre l'unité abstraite du « genre » et la multiplicité de ses réalisations. Il mentionne dans l'ordre : a) la sculpture ; b) la peinture ; c) la poésie dramatique latine et grecque ; d) l'éloquence grecque et latine (\$ 26-28). La correspondance avec le schéma du Brutus s'explique par la hiérarchie des arts dans le monde ancien : a) arts plastiques et figuratifs, qui dépendent du travail manuel et de l'évidence sensible ; b) arts de la parole, avec poésie dramatique et poésie épique en ordre fluctuant. Cicéron, qui partage cette conception, assigne toutefois la primauté à l'éloquence. Cf. Marino BARCHIESI, Nevio epico. Storia, interpretazione, edizione critica dei frammenti del primo epos latino, Padova, Cedam, 1962, pp. 30-38 ; Ernst Hans Gombrich, «The Debate on Primitivism in Ancient Rhetoric », Journal of the Warburg and Courtauld Institutes 29, pp. 24-38: 28-30 ; Douglas, « The Intellectual Background », art. cit., pp. 108-115. 
illusion de solidité par le moyen bidimensionnel du tableau ${ }^{41}$, Apelle et Protogène - chez qui iam perfecta sunt omnia (\$ 70) - poursuivaient le même effort que fut celui qui avait amené Polyclète à se démarquer de ses prédécesseurs, en réalisant des statues plane perfecta, représentant admirablement la ueritas ou naturalité des choses ${ }^{42}$. Dans le cas d'Aristophane de Byzance, son attitude normative pourrait donc être interprétée comme une rupture à l'égard de l'usage pour améliorer la manière dont les mots rendent leurs désignations.

Dans la suite de son argumentation Varron précise que, au-delà des situations où se manifeste une urgence, les locuteurs doivent employer les formes analogiques, en restaurant les anciennes non encore corrompues ou en en adoptant de nouvelles qui soient conformes à la ratio. Consuetudo loquendi est in motu ( 17 ), affirme-t-il, et c'est aux poètes, notamment aux poètes dramatiques, qu'il revient d'habituer les locuteurs aux innovations, la langue poétique étant conçue comme un système autonome, pourvoyeur de valeurs esthétiques et formelles qui peuvent devenir indépendantes du texte-source pour être progressivement transférées dans la langue de la conversation ordinaire. Les orateurs en revanche jouent un rôle passif à l'égard de la consuetudo, car ils sont obligés de la respecter soigneusement pour pouvoir se faire comprendre et pour mettre l'auditoire en de bonnes dispositions d'écoute ${ }^{43}$.

Ces considérations montrent clairement que Cicéron et Varron partagent la même vision dynamique de la langue, soumise à un processus de déviation par rapport à un état de perfection où la transparence des mots correspondait à leur insertion parfaite dans un système de rapports rationnels. Cela étant, opposant deux ueritates ou principes propres à deux disciplines distinctes, Cicéron place l'efficacité persuasive au-dessus de la lisibilité des formes qui respectent l'analogie ; Varron, en revanche, affirme sa volonté de systématiser l'analogie, aussi inusuelle soit-elle, en lui restituant le rôle de principe universel, dont l'application s'étend bien au-delà de la grammaire ${ }^{44}$. Comme le laisse supposer la mention d'Aristophane en lien avec les peintres les plus connus pour leur

41. Cf. Alan E. Douglas, M. Tulli Ciceronis Brutus, Oxford, Clarendon, 1966, ad loc.

42. Cf. Cicéron Brut. 70.

43. Deux autres taxinomies des locuteurs figurent en ling. 9,114 et 10,74, où l'orator est remplacé par les singuli e populo. Sur ces textes très étudiés, cf. notamment Elmar SiEBENBORn, Die Lehre von der Sprachrichtigkeit und ihren Kriterien. Studien zur antiken normativen Grammatik, Amsterdam, Grüner, 1976, pp. 113-114 ; GARBARINO, «Verba poetica », art. cit., pp. 181-183; Valeria LomAnTo, «Cesare e la teoria dell'eloquenza », Memorie dell'Accademia delle Scienze di Torino 18-19, 1994-1995, p. 3-127 : 78-95. Sur le rapport entre éloquence et poésie, cf. GARBARINO, «Verba poetica », art. cit., pp. 146-152. 164-176; sur la catégorie des poètes chez Varron : Giorgio PIRAS, Varrone e $i$ poetica uerba. Studio sul settimo libro del de lingua Latina, Bologna, Pàtron, 1998, pp. 94-105 ; Jacqueline DANGEL, «Varron et les citations poétiques dans le De lingua Latina », in Gualtiero Calboli (ed.), Papers on Grammar, 6, Bologna, Clueb, pp. 97-122.

44. Pace P. WÜLfING VON MARTITZ, «Verus, uerum und ueritas », Glotta 46, 1968, pp. 278-293 : 286 ; RISSANEN, « Some Aspects », art. cit., p. 122 et n. 13. 
illusionnisme réaliste, cette ratio se fonde sur l'idée d'un rendu fidèle, de la part des propriétés formelles des mots, des caractéristiques sémantico-référentielles des denotata ${ }^{45}$ : traduisant fidèlement l'univers qu'elle est censée renfermer, la ueritas linguistique de Varron récupère la puissance gnoséologique de l'alêtheia.

Ainsi, dans le cas des terminaisons parallèles du génitif des thèmes en -oqui figurent également dans l'excursus de l'Orator cicéronien, Varron réfute les critiques des anomalistes ${ }^{46}$ en faisant découler le choix entre -um et -orum de la dichotomie entre déterminé et indéterminé. L'application récursive de ce critère, utilisé par Chrysippe dans le cadre d'une division ontologique entre la qualité propre désignée par l'onoma et la qualité commune présupposée par la prosêgoria ${ }^{47}$, s'étend ici aux référents unitaires ou multiples auxquels renvoient respectivement les dénombrables et les collectifs. La terminaison -orum devrait donc s'appliquer aux seuls référents indéterminés, la terminaison -um, aux déterminés (ling. 9,85) :

« Le déterminé [finitum] et l'indéterminé [infinitum] étant différents, il ne faut pas - me semble-t-il - les exprimer de la même manière, d'autant plus que quand on ajoute un nombre défini de milliers à ces mots, on emploie une forme différente de celle qu'on emploie normalement. En effet, on dit au singulier mille denarium, non mille denariorum, et au pluriel duo milia denarium, non duo milia denariorum. Si l'on prenait le nominatif pluriel denarii, désignant une quantité indéterminée, on emploierait la forme du génitif denariorum. Et la même chose doit être remarquée non seulement dans les mots denarii, uictoriati, drachmae, nummi, mais aussi dans uiri : quand nous disons qu'il y a eu une délibération des triumuiri, des decemuiri, des centumuiri, nous employons les formes triumuirum, decemuirum, centumuirum, non triumuirorum, decemuirorum, centumuirorum.»

Cette conciliation entre les deux vérités - l'une technique, l'autre universelle - permet de souligner la fonction gnoséologique du langage, ainsi que le montrent notamment les livres étymologiques du De lingua Latina qui, sous prétexte d'enquêter sur l'origine des mots, offrent une vue panoramique des mœurs et des institutions archaïques de Rome suivant les catégories extralinguistiques de locus, corpus, tempus, actio, combinées avec une ontologie de dérivation stoïcienne ${ }^{48}$. Mais cette même conciliation constitue aussi la limite

45. Cf. Siebenborn, Die Lehre, op. cit., pp. 113-114.

46. Cf. Varron ling. 8,71.

47. Cf. Varron ling. 8,80; Raffaella PETRILLI, Temps et détermination dans la grammaire et la philosophie anciennes, Münster, Nodus, 1997, pp. 176-177.

48. Pour une présentation d'ensemble, cf. notamment PIRAs, Varrone, op. cit., pp. 127-143. La question des influences philosophiques du De lingua Latina est extrêmement complexe. L'apport essentiel du Portique est quand même indéniable, car Varron lui-même déclare s'inspirer à la fois 
fondamentale de la proposition théorique de Varron, car elle empêche la science linguistique de se rendre indépendante en tant que savoir délimité et dès lors " auto-nome », la ratio propre à celle-ci étant vue non comme un outil créé par l'homme, dont la rationalité se manifeste dans une relation fin/moyens, mais comme une espèce du genre universel de la raison cosmique, d'un principe explicatif immanent au monde.

Notre étude a mis en évidence deux perspectives fondamentales qui soustendent deux disciplines naissantes à la fin de la République à Rome. L'une, intralinguistique et « auto-nome », concerne le système que l'on peut concevoir par convention à partir de l'ensemble des procédés de vérification établissant la correction ou la conformité à ce même système: dans ce cas, la vérité correspond au principe de fonctionnement de l'analogie linguistique, telle qu'on la trouve aussi bien dans l'excursus de l'Orator cicéronien que dans le De lingua Latina de Varron. L'autre, extralinguistique, et par conséquent hétéronome, amène Cicéron à concevoir la théorie du uerisimile de l'éloquence, dont l'efficacité pragmatique peut revendiquer la force persuasive des choses elles-mêmes, de la réalité dans son évidence sensible ; dans le cas de Varron, la reconstitution d'un système totalisant comme celui du langage gouverné par l'analogie aboutit à l'idée d'obliger la nature tout entière à se dévoiler, à parvenir à une vérité universelle qui, d'élément latent, passe à l'état de déploiement d'elle-même via l'analyse minutieuse des mots.

Alessandro GARCEA

Université de Toulouse 2 \& UMR 7597

de sources stö̈ciennes et grammaticales: non solum ad Aristophanis lucernam, sed etiam ad Cleant<i>s lucubraui (ling. 5,9); auctor [...] mihi Chrysippus et Antipater et [...] Aristophanes et Apollodorus (ling. 6,2). Pour un état des lieux, cf. Franco CAVAZZA, Studio su Varrone etimologo e grammatico, Firenze, La Nuova Italia, 1981, pp. 46-54. Il est fort probable qu'Antiochus d'Ascalon a joué le rôle de passeur : cf. Alain MicheL, «Le philosophe, le roi et le poète dans le De lingua Latina », Revue de Philologie 39, 1965, pp. 69-79. Sur l'importance de natura et usus dans le livre 9 du De lingua Latina, cf. Wolfram Ax, «Pragmatic Arguments in Morphology: Varro's Defence of Analogy in Book 9 of his De Lingua Latina », in Pierre Swiggers \& Alfons Wouters (eds.), Ancient Grammar : Content and Context, Leuven, Peeters, 1996, pp. 105-119. 